\title{
The design of automatic patients nursing system based on computer vision
}

\author{
Zhangting \\ Zaozhuang Vocational College of Science\&Technology shandong, tengzhou, 277500
}

Keywords: computer vision; nursing; patients

\begin{abstract}
The design of automatic patients nursing is researched. The behavior of patient have bigger burst and random, and blind spot exists in nursing. The traditional nursing system design method is lack of recognition ability for independent behavior of patient, unable to carry out alarming for patient in sudden situation. For this reason, an automatic patients nursing system design method based on catfish particle swarm optimized neural network algorithm is proposed. The BP neural network model was established, by using particle swarm optimization algorithm to optimize BP neural parameters, and "catfish effect" is introduced to overcome the local optimum defects of particle swarm optimization algorithm, with the specific patient image database to do simulation test for the performance of the algorithm. The simulation results show that, compared with the traditional design method of patient nursing system, CFPOS-BP can obtain better rationality of automatic patient nursing, not only improve the patient image recognition accuracy and efficiency, and solve the optimization problem of BP neural network parameters.
\end{abstract}

\section{Introduction}

With the increasingly tense of medical resources, automatic patient nursing has become a nursing method worth being advocated [1]. The artificial intelligence method is applied to care patients to ensure safe and comfortable life for patients [2]. Therefore, design methods for automatic patient nursing system, have become a hotspot problems need to be studied in the medical field [3]. At this stage, the main design methods for automatic patient nursing system include design method for automatic patient nursing system based on genetic algorithm, support vector machine algorithm and artificial immune algorithm [4-6]. Among them, the most commonly used is the design method for automatic patient nursing system based on artificial immune algorithm [7]. Because design methods for automatic patient nursing system have extremely broad space for developing in medical field, therefore, received a lot of attention from the experts.

The behavior of patient have bigger burst and random, and blind spot exists in nursing. The traditional nursing system design method is lack of recognition ability for independent behavior of patient, unable to carry out alarming for patient in sudden situation.

In order to avoid the defect of the algorithm described above, an automatic patients nursing system design method based on catfish particle swarm optimized neural network algorithm is proposed. The BP neural network model was established, by using particle swarm optimization algorithm to optimize BP neural parameters, and "catfish effect" is introduced to overcome the local optimum defects of particle swarm optimization algorithm, with the specific patient image database to do simulation test for the performance of the algorithm. The simulation results show that, compared with the traditional design method of patient nursing system, CFPOS-BP can obtain better rationality of automatic patient nursing, not only improve the patient image recognition accuracy and efficiency, and solve the optimization problem of BP neural network parameters.

\section{The design principle of automatic patients nursing system}

According to the design principle of automatic patient nursing system, to complete automatic patients nursing system, so in shortage of medical resources, can provide maximal nursing to the patient, and ensure the safety of patients. Its principle is as follows: 
The elements in the immune set of nursing patient images can be depicted by $T=\prod_{j=1}^{\infty}\{0,1\}^{j}$, the corresponding characteristic can be represented the following formula:

$$
\left\{\begin{array}{c}
T_{k} \subset T \\
B=\left\{\langle b, c\rangle|b \in E \cup| b \mid=m, c \in T_{k}\right\} \\
E=\{0,1\}^{m}
\end{array}\right.
$$

In the formula, $|b|$ can be used to describe the number of elements in immune collection of patient nursing image.

During the setting process of automatic patients nursing system design, antigen sets can be described by $B^{\prime}$, and $B^{\prime} \subset B$, then in view of the above elements, the initial patient nursing image data packet is $\left\langle B_{j}^{\prime}, c\right\rangle$, the corresponding characteristic can be described with the following formula:

$$
\left\langle B_{j}^{\prime}, b\right\rangle=A P C t\left\langle B_{j}^{\prime}, c\right\rangle
$$

During the setting process of automatic patients nursing, the collection constructed of autoimmune can be described by $T G$, corresponding non self sets can be described by $N T G$, and $B=\langle T G, N T G\rangle$.

The following formula is adopted to calculate the affinity between antigen and antibody during the automatic patient nursing process:

$$
g_{\text {Match }}\left(B_{j}^{\prime}, B_{k}^{\prime}\right)=\operatorname{sim}\left(B_{j}^{\prime}, B_{k}^{\prime}\right)=\frac{\sum_{j=1}^{M} \sum_{k=1}^{G}\left|B_{j}^{\prime} \cap B_{k}^{\prime}\right|}{\sqrt{\sum_{j=1}^{M}\left|B_{j}^{\prime}\right|^{2}}+\sqrt{\sum_{k=1}^{G}\left|B_{k}^{\prime}\right|^{2}}}
$$

In the formula, $B_{j}^{\prime}$ can be used to describe the number of patient behavior in antigen random sequence value of $j$-th function in certain time period, $B_{k}^{\prime}$ is the number of corresponding patient behavior of autologous scattered sequence values, $\sum_{j=1}^{M} \sum_{k=1}^{G}\left|B_{j}^{\prime} \cap B_{k}^{\prime}\right|$ is the hash value when antigen and antibody in the immune collection applied to the specified patient behavior image. $\sum_{j=1}^{M}\left|B_{j}^{\prime}\right|$ can be used to describe the number of patient behavior characteristics when antigens in the time domain, $\sum_{j=1}^{M}\left|B_{k}^{\prime}\right|$ can be used to describe the number of patient behavior characteristics when corresponding antibodies in time domain.

The threshold of patient behavior characteristics can be described with $\chi$. According to the behavior antibodies of all patients, the following formula is needed for judging if the behavior is abnormal:

$$
\begin{aligned}
& e_{j}=\sum_{k=1}^{p} \frac{\left\|B_{k}^{\prime}-B_{j}^{\prime}\right\| * f^{-\left\|B_{k}^{\prime}-B_{j}^{\prime}\right\|}}{\sum_{k=1}^{p} f^{-\left\|B_{k}^{\prime}-B_{j}^{\prime}\right\|}} \\
& \chi=\frac{q^{\frac{1}{2}}}{p}\left(\sum_{j=1}^{p} e_{j}\right)
\end{aligned}
$$

According to the method described above, the automatic patient nursing system design can be completed, so as to guarantee the patient's safe in the process of medical treatment. However, the behavior of patient have bigger burst and random, and blind spot exists in nursing, unable to carry out alarming for patient in sudden situation.

\section{Design optimization method of automatic patients nursing system}

The design of automatic patients nursing system using the traditional algorithm, since the behavior of patient have bigger burst and random, and blind spot exists in nursing, unable to carry out 
alarming for patient in sudden situation. For this, an automatic patients nursing system design method based on catfish particle swarm optimization neural network algorithm.

The catfish particle swarm algorithm based on computer vision image for patients nursing. According to the "catfish effect" enlightenment: when the patient nursing computer vision image particle gathered in the local optimum and caused search stagnation, a "catfish" needs to be found to stimulate the patient's nursing image particle swarm, and change the aggregation state of particle swarm in local optimal location, so as to make the particle swarm out of local minimum point and find the global optimum, this is the basic idea of the patient nursing computer vision image catfish PSO (CFPSO). CFPSO uses the deviation threshold as a trigger condition, through catfish operator to disturb the global extremum or individual extremum, patient nursing computer vision image particle velocity is updated as follows:

$$
\begin{aligned}
v_{i, d}^{k+1}= & \omega \cdot v_{i, d}^{k}+c_{1} \cdot \operatorname{rand}() \cdot\left(c_{3} \cdot \operatorname{rand}() \cdot \text { pbest }_{i, d}^{k}-x_{i, d}^{k}\right) \\
& +c_{2} \cdot \operatorname{rand}() \cdot\left(c_{4} \cdot \operatorname{rand}() \cdot \operatorname{gbest}_{d}^{k}-x_{i, d}^{k}\right)
\end{aligned}
$$

In the formula, $\tilde{x}_{i j}=x_{\max }+x_{\min }-x_{i j}$ represents the collision intensity of catfish on optimum individual of patient nursing computer vision image, $c_{4}$ is the collision intensity of catfish on global optimal; $c_{3} \cdot \operatorname{rand}()$ and $c_{4} \cdot \operatorname{rand}()$ are called catfish operator, which are defined as follows:

$$
\begin{aligned}
c_{3} \cdot \operatorname{rand}() & = \begin{cases}1, & e_{p}>e_{0 p} \\
c_{3} \cdot \operatorname{rand}(), & e_{p} \leq e_{0 p}\end{cases} \\
c_{4} \cdot \operatorname{rand}() & = \begin{cases}1, & e_{g}>e_{0 g} \\
c_{4} \cdot \operatorname{rand}(), & e_{g} \leq e_{0 g}\end{cases}
\end{aligned}
$$

In the formula, $e_{p}$ represents the deviation of the current value and current optimal individual value; $e_{g}$ represents the deviation of the current value and current global optimal value; $e_{0 p}$ represents the threshold of the deviation between the current value and current local optimal value; $e_{0 g}$ represents the threshold of the deviation between the current value and the current global optimal value.

Based on the above formula, if the deviation of the current value is greater than deviation threshold, catfish operator is 1, and the CFPSO algorithm is the standard PSO algorithm; on the contrary, the particle aggregation occurs, catfish operator is introduced to collide with individual optimal values or global optimal value, so as to avoid local optimal.

Catfish particle swarm algorithm for optimizing patient computer vision image BP neural network.The detailed design steps to realize automatic patients nursing system are as follows:

(1) initialize the patient computer vision image BP neural network, set the neuron number in input layer, hidden layer, output layer of network, transfer function, and learn step size etc..

(2) Randomly initialize the velocity of patient computer vision image particle swarm and each particle, size of population, the inertia weight, learning factor, the iteration number.

(3) transfer the initial position of patient computer vision image particles to BP neural network as the initial weights and threshold of patient computer vision image BP neural network. The samples is input into network, according to forward calculation method of BP neural network to calculate the network output value.

(4) Calculate the error of patient computer vision image BP neural network, and the network error is returned to the particle swarm algorithm as the algorithm's fitness.

(5) Compare the current fitness value and individual optimal fitness value of each patient computer vision image particle, if the current fitness is better, then the current fitness value is viewed as individual optimal fitness, and record the position of the particles as the individual optimal position.

(6) Compare the current fitness value and global optimal fitness value of all patients computer vision image particle, if the current fitness is better, then the current fitness value is viewed as global optimal fitness, and record the position of the particles as the global optimal position.

(7) determine the patient computer vision image catfish operator, and updates the particle velocity and position. 
(8) if the number of iterations exceeds the maximum allowed number of iterations, then terminate the training.

(9) the output global optimal position output is the best weights and the optimal threshold of patients computer vision image neural network. Otherwise, return to step (4), the algorithm continues iterating.

\section{Experiment results and analysis}

In order to verify the superiority of the proposed automatic patient nursing system design method, there is the need for an experiment. During the process of the experiment, from the images collected with the sensor, randomly selected 4 frames of patients behavior image, the image is as follows:

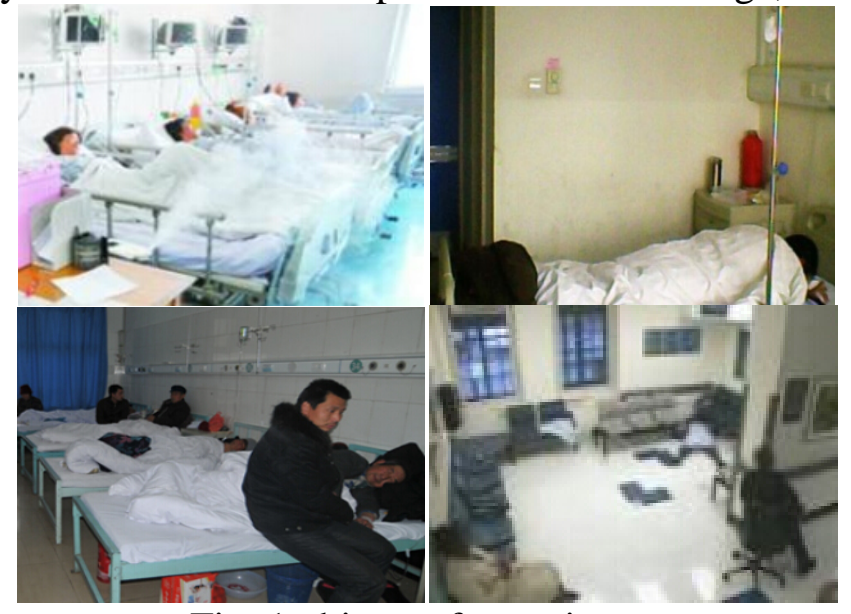

Fig. 1 objects of experiment

With traditional algorithms to do automatic patients nursing, obtained results can be described with the figure below:

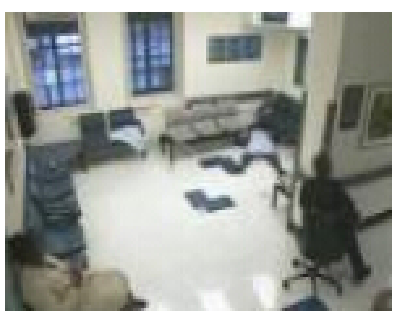

Fig. 2 experimental results obtained with traditional algorithm

With the proposed algorithm to do automatic patients nursing, obtained results can be described with the figure below:

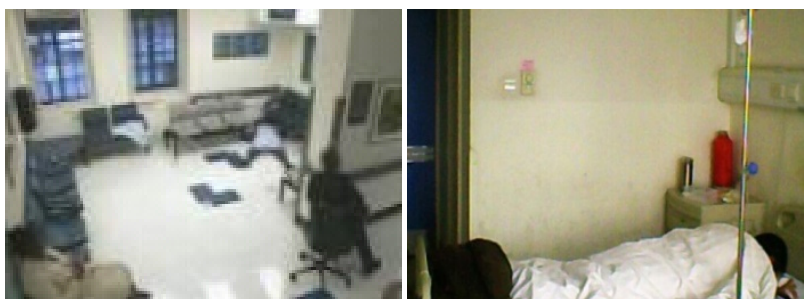

Fig. 3 experimental results obtained with proposed algorithm

1000 images of patients were collected, respectively, using different algorithms for automatic patient nursing, the following results was obtained: 
Table 1 The experimental data table obtained with different algorithms

\begin{tabular}{lll}
\hline \multirow{2}{*}{$\begin{array}{l}\text { The number } \\
\text { experiments }\end{array}$} & \multirow{3}{l}{ of Identification error rate $(\%)$} \\
\cline { 2 - 3 } & $\begin{array}{l}\text { The traditional } \\
\text { algorithm }\end{array}$ & $\begin{array}{l}\text { The proposed } \\
\text { algorithm }\end{array}$ \\
\hline 1 & 9 & 13 \\
2 & 9 & 11 \\
3 & 8 & 12 \\
4 & 7 & 14 \\
5 & 8 & 13 \\
6 & 8 & 14 \\
7 & 9 & 11 \\
8 & 7 & 12 \\
9 & 9 & 14 \\
10 & 6 & 16 \\
\hline
\end{tabular}

Based on the data in the table, the proposed algorithm can effectively improve the recognition rate of the patients in unexpected situation, so as to guarantee the safety of patients.

\section{Conclusions}

An automatic patients nursing system design method based on catfish particle swarm optimized neural network algorithm is proposed in this paper. The BP neural network model was established, by using particle swarm optimization algorithm to optimize BP neural parameters, and "catfish effect" is introduced to overcome the local optimum defects of particle swarm optimization algorithm, with the specific patient image database to do simulation test for the performance of the algorithm. The simulation results show that, compared with the traditional design method of patient nursing system, CFPOS-BP can obtain better rationality of automatic patient nursing, not only improve the patient image recognition accuracy and efficiency, and solve the optimization problem of BP neural network parameters.

\section{References}

[1] You Ting, Li Peijiang. Based on Wireless Networks Sewage Monitoring System Research [J]. Computer Simulation,2012.2:94-97.

[2] Mi Xiaoping, Li Xuemei. Old People Who Live Alone Automatic Monitoring Method Based on Iot Intelligence Research [J]. Computer Simulation,2014.2:378-381.

[3] Han Lei, Li Junfeng, Jia Yunde. Human Interaction Recognition Using Spatio-Temporal Words [J].Chinese journal of computers, 2010.4:776-784.

[4] Li Ning, Xu De, Fu Xiaoying, Yuan Ling. Action Recognition Combined with Human Action Property [J]. Journal of Beijing Jiaotong University: Natural Science Edition, 2009.2:6-10.

[5] Zhu Xudong, Liu Zhijing. Human abnormal behavior identification based on theme Hidden Markov model [J].Computer Science, 2012.3:251-255.

[6] Hong Yunguo. Human behavior recognition model based on improved Canny operator and Neural Network [J].Computer engineering and application, 2013.8:156-159.

[7] Mei Yi, Sun Hongwei. Simulation of cheating behavior recognition based on intelligent vision [J]. Computer Simulation,2014.4:219-222. 